\title{
Recruitment of the ventral and dorsal streams in statistical graph comprehension: An fMRI study
}

\author{
Mi Li ${ }^{\mathrm{a}, \mathrm{b}, \mathrm{c}}$, Shengfu Lu ${ }^{\mathrm{a}, \mathrm{b}, \mathrm{c}, *}$ and Ning Zhong ${ }^{\mathrm{a}, \mathrm{b}, \mathrm{c}, \mathrm{d}}$ \\ ${ }^{a}$ The International WIC Institute, Beijing University of Technology, Beijing, China \\ ${ }^{\mathrm{b}}$ The Beijing International Collaboration Base on Brain Informatics and Wisdom Services, Beijing, \\ China \\ ${ }^{\mathrm{c}}$ The Beijing Key Laboratory of MRI and Brain Informatics, Beijing, China \\ ${ }^{\mathrm{d}}$ The Department of Life Science and Informatics, Maebashi Institute of Technology, Maebashi-City \\ Maebashi, Japan
}

\begin{abstract}
.
BACKGROUND: Although many previous studies have focused on statistical graph comprehension in cognitive psychology, there is no consensus among them.

OBJECTIVE: Brain neuroimaging studies on the statistical graph comprehension are useful to account for the cognitive mechanism of interpreting statistical graphic information.

METHODS: The present study used two experimental conditions, a statistical graph (SG) and a statistical graph with text (SGT), and one control condition, a text (ST), where the ST task was a verbal description of the information from the SG, and when the SGT is a mixed graph + textual description. We used fMRI to investigate the brain activity of 36 normal subjects while they passively viewed the statistical information presented in visual forms as SG, ST or SGT.

RESULTS: The results showed that compared with the control tasks, both SG and SGT consistently activated ventral and dorsal streams and that compared with SGT, SG significantly activated the ventral stream but not the dorsal stream.

CONCLUSIONS: These results suggest that the portions of the ventral and dorsal streams related to object recognition are commonly involved in statistical graph comprehension. These findings provide neuroimaging evidence for the cognitive processing of statistical graphs.
\end{abstract}

Keywords: fMRI, statistical graph, statistical graph with text, ventral stream, dorsal stream

\section{Introduction}

Statistical graphs are widely used to represent quantitative data in science, education, and business, which are an important part of statistical data analysis. Such graphs are considered a vital information carrier because they facilitate easy remembering and comprehension. In general, statistical graphs (e.g., bar or line graphs) consist of basic elements such as the framework, labels, and specifiers [1|2]. The framework is divided into two parts: an external framework (X, Y coordinates) and an internal framework

\footnotetext{
${ }^{*}$ Corresponding author: Shengfu Lu, The International WIC Institute, Beijing University of Technology, Ping Le Yuan 100, Chaoyang District, Beijing 100124, China. Tel.: +86 106739 6464; Fax: +86 106739 6295; E-mail: lusf@ bjut.edu.cn.
} 
(horizontal or vertical grid lines). The label is an explanation of a bar or a region using letters, words or numbers, (e.g., X, Y-axis scales), and the specifier is the entity (e.g., the data point, bar or line) in a statistical graph.

In cognitive psychology, some studies have mainly concentrated on perceptual aspects in statistical graph comprehension, such as reading off a y-value from a bar or line graph; or extracting a trend from a line graph [3]. More recent studies have begun to argue that graph comprehension, in reality, is hierarchical; first is a reading of the explicitly represented data; and then interpreting information, which involves spatial processing, such as maintaining the location information of each entity (e.g., the relative location of each bar), or spatial transformation for comparison among entities (e.g., the mental operation during transforming a bar from one location into another) [4[5]. A more difficult question is integration, where multiple data points need to be extracted and integrated by some mental operations because the information is not explicitly represented in the graph. Therefore, Trickett and Trafton [6] have proposed that spatial processing is involved in a range of graph tasks (even only reading a y-value of a bar or comparing between two adjacent bars), regardless of the complexity of the graphs. Taken together, the perceptual theory of graph comprehension and the hierarchical theory use cognitive behavior to explain graph comprehension tasks; however, no consensuses exsits. This present study, using a neuroimaging perspective, may provide some evidence for this issue.

In object recognition, there are two segregated pathways in the human brain: a ventral stream and a dorsal stream. The ventral stream runs from the occipital lobe to the inferotemporal cortex, and the dorsal stream runs from the occipital lobe to the posterior parietal lobe. A large number of studies have shown that the ventral stream is needed to identify the characteristics of objects (such as color, shape, and size), whereas the dorsal stream is recruited more for the processing object spatial properties [7/8]. Lesion studies suggest that damage to the ventral stream leads to impairment in object recognition, and lesions to the dorsal stream lead to impairment in spatial processing [7]9]. Specifically, lesions in the occipitotemporal region of visual cortex often lead to object recognition impairments [8].

According to statistical graphs characteristics, it is reasonable to suppose that statistical graph comprehension would activate the ventral and dorsal streams, which are involved in object recognition. In statistical graphs, spatial characteristics are used to represent the quantitative relationships of various objects [1] because statistical graphs are based on the location relationship to describe the associations between an object's name and value as well as the order relationship between objects. Thus, during statistical graph comprehension, subjects often need to identify the elements, such as the entities (e.g., Bar or Line) and labels. This cognitive processing is similar to object recognition [7]8], which may require the involvement of the ventral stream. Additionally, statistical graph comprehension also involves spatial processing, such as identifying the locations of the entities or labels, and their spatial relationships [4], which are likely derived from objects' spatial-location recognition or spatial analysis, and may require the involvement of the dorsal stream.

To investigate this issue, the present study used two experimental conditions, a statistical graph (SG) and a statistical graph with text (SGT), and one control condition, a text (ST), where the ST task was a verbal description of the information in the SG, and the SGT was a mixed graph + textual description. In our study, SGT tasks were used to distinguish between the brain regions involved in perceptual processing and those tasks involved in spatial processing during statistical graph comprehension. Previous studies showed that during verification of text-graphical tasks, subjects read the text before viewing the graphics [10]. Thus, SGT comprehension is text-oriented [11]. The SGT text in our study is mainly a verbal description of the items on the graphs, which do not involve information analysis (e.g., predicting a trend). Therefore, SGT text only affects the perceptual operation for extracting information but has 


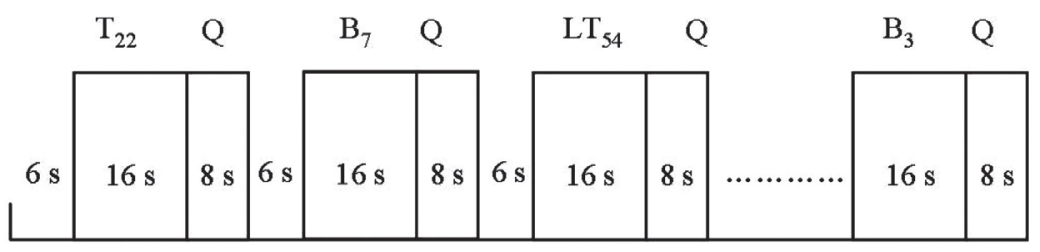

Fig. 1. The paradigm of stimuli presentation in a session. T, a text that is a control task; B, a bar-graph task; LT, a line graph with text task; Q, a question.

little effect on spatial analysis. Although both SG and SGT involve perceptual processing while subjects are extracting information, these two cognitive processes are different. For SG, the process of extracting information is based mainly on visual features (e.g., entities [Bar or Line], coordinates and labels); for SGT, subjects first obtain the data from the text and then verify the data on the graphs. Thus, during SG comprehension, the brain regions related to graphical perceptual processing respond and should be stronger compared with SGT, perhaps reaching statistical significance. In contrast, SG and SGT should not produce significant response differences in the brain regions related to spatial-location recognition or spatial analysis.

\section{Materials and methods}

\subsection{Participants}

Thirty-six subjects ( 18 women and 18 men, mean age $22.5, \mathrm{SD}=1.7$ ) were recruited from the Beijing University of Technology. All were right handed and had normal hearing and normal (corrected) vision. The study was approved by the institutional Review Board of the Beijing University of Technology, and also by the Ethical Committee of Xuanwu Hospital of the Capital Medical University.

\subsection{Experimental stimuli and design}

The experiment included two experimental conditions: a statistical graph (SG) and a statistical graph with text (SGT), and one control condition: a text (ST), in which the ST task is a verbal description of the information from the SG, and the SGT is a mixed graph + textual description. Each condition included 20 stimuli. The 60 stimuli were divided into three groups on average. The order of these tasks was pseudo randomized in each group. Each stimulus was presented for a period of $16 \mathrm{~s}$. The paradigm of stimuli presentation in a session was shown in Fig. 1.

\section{3. fMRI data acquisition}

Scans were acquired on a 3-Tesla scanner (Trio system) using spiral acquisition with a standard head coil. T1-weighted scans ( 30 slices, inter-slice interval $=0.8 \mathrm{~mm}$, slice thickness $=4 \mathrm{~mm}, \mathrm{TR}=130 \mathrm{~ms}$, $\mathrm{TE}=2.89 \mathrm{~ms}, \mathrm{FA}=70^{\circ}, \mathrm{FOV}=240 \times 240$, Voxel $=0.8 \times 0.8 \times 4 \mathrm{~mm}^{3}$, the matrix size $=320$ $\times 320$ ) were acquired and averaged to provide high-resolution images on which to identify anatomical landmarks. Functional scans (30 slices, inter-slice interval $=0.8 \mathrm{~mm}$, slice thickness $=4 \mathrm{~mm}$, TR $=$ $2000 \mathrm{~ms}, \mathrm{TE}=31 \mathrm{~ms}, \mathrm{FA}=90^{\circ}, \mathrm{FOV}=240 \times 240$, Voxel $=4 \times 4 \times 4 \mathrm{~mm}^{3}$, the matrix size $=64 \times$ $64)$. 


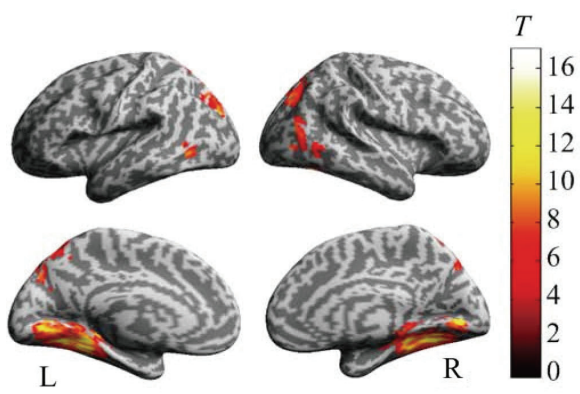

(a) SG vs. ST

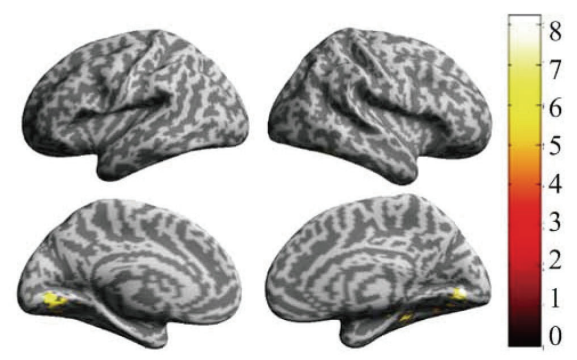

(c) SG vs. SGT

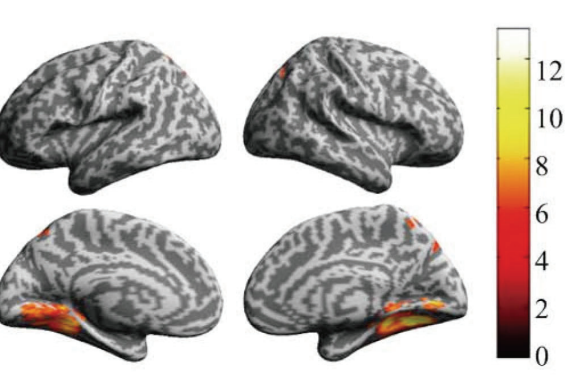

(b) SGT vs. ST

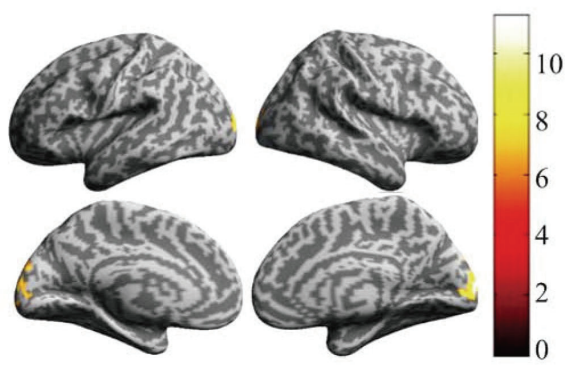

(d) SGT vs. SG

Fig. 2. Whole-brain statistical activation maps directly comparing between the tasks: (a) SG vs. ST, the contrast showed significant activation in the ventral and dorsal streams; (b) SGT vs. ST, the contrast showed significant activation in the ventral and dorsal streams; (c) SG vs. SGT, the contrast showed that only the ventral stream was more significant activated. (d) SGT vs. SG, the contrast showed only the striate cortex/prestriate cortex (BA17/18), no activation in dorsal stream. All of the Statistical Parametric Mapping t of the contrasts was thresholded at (a), (b) $p<0.05$, corrected, cluster size $\leqslant 400 \mathrm{~mm}^{3}$; (c), (d) $p<$ 0.001 , uncorrected, cluster size $\leqslant 400 \mathrm{~mm}^{3}$.

\subsection{Preprocessing and fMRI data analysis}

Data were statistically analyzed by using SPM8. Images were slice-time corrected, realigned, coregistered, normalized, and smoothed according to standard pre-processing methods. The data underwent high-pass filtering to consider low-frequency drift, with a cut-off value of 128. An auto-regression AR (1) was used to exclude the variance explained by the pervious scan. The least squares parameter estimates of the height of the best fitting synthetic hemodynamic response function (hrf) were used in the first level statistical analysis (single subject) for each condition in pairwise contrasts. A random effect model was used in the second level statistical analysis (group analysis). The images were then tested against the null hypothesis. In the group-level, one-sample t-tests were implemented for each voxel of the contrast images. In this study, a region was considered significant if exceeds an alpha threshold of $(p<0.05$, corrected) and $(p<0.001$, uncorrected). Regions of activation originally obtained in MNI coordinates were converted into Talairach coordinates.

\section{3. fMRI results}

\section{1. $S G v s . S T$}

As shown in Fig. 2(a), the SG compared with the control tasks (ST) more significantly activated the ventral and dorsal streams, in which the ventral stream had a $26224 \mathrm{~mm}^{3}$ cluster size including the 


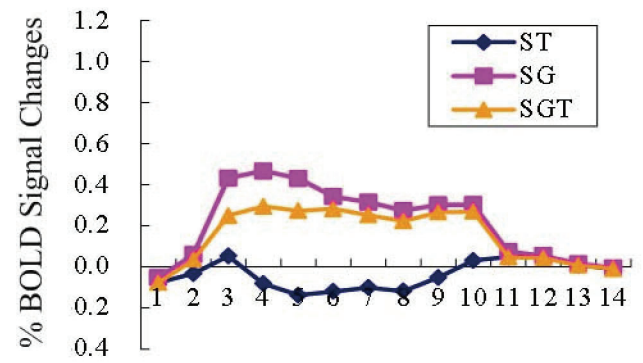

(a) Left Dorsal stream

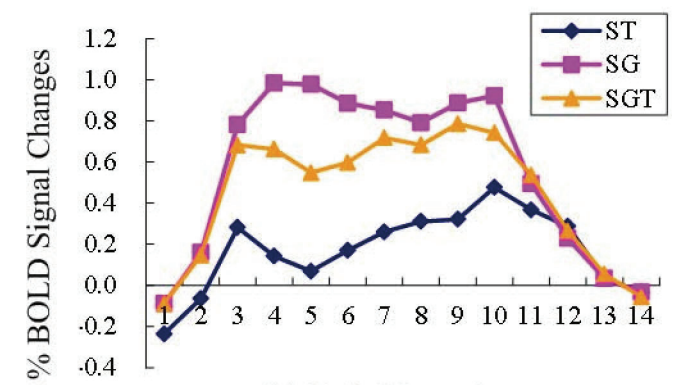

(c) Left Ventral stream

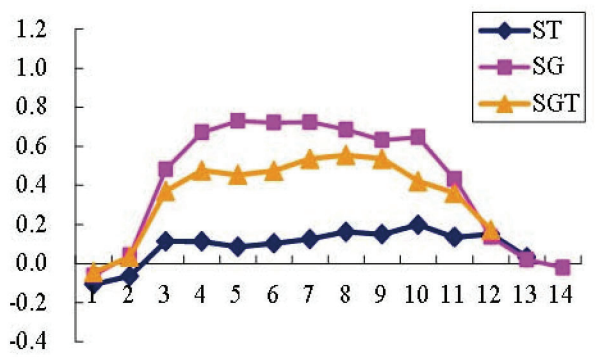

(b) Right Dorsal stream

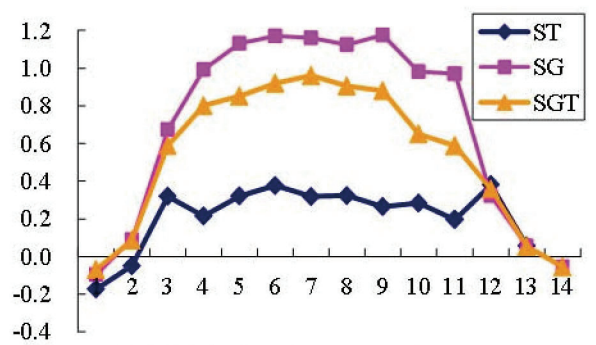

(d) Right Ventral stream

Fig. 3. For each subject, separate ROI analysis was conducted within dorsal stream and ventral stream regions of left and right (here shown averaged across subjects). (a) left dorsal stream, (b) right dorsal stream, (c) left ventral stream, (d) right ventral stream; SG: statistical graph; ST: text; SGT: statistical graph with text.

bilateral lingual gyrus [BA18, Talairach (TAL): $-8 /-74 / 2, t=12.01 ; 8 /-72 / 7, t=11.08]$, fusiform gyrus (BA19, TAL: $-26 /-68 /-5, t=16.10 ; 26 /-68 /-5, t=12.12$ ), posterior parahippocampal gyrus (BA19/37, TAL: $-32 /-43 /-8, t=20.10 ; 30 /-55 /-9, t=13.34$ ), and middle temporal gyrus (BA19, TAL: $-40 /-83 / 19, t=8.95 ; 42 /-81 / 19, t=6.80)$; the dorsal stream had a $8456 \mathrm{~mm}^{3}$ cluster size including the bilateral superior occipital gyrus (BA19, TAL: $-32 /-82 / 24, t=9.55 ; 34 /-78 / 30, t=$ 7.40), left precuneus (BA7, TAL: $-14 /-68 / 44, t=7.74$ ), and right superior parietal lobule extending to the precuneus (BA7, TAL: $28 /-66 / 46, t=6.98$ ).

The blood oxygenation level-dependent (BOLD) signal changes in peak voxel in the dorsal stream and ventral stream of left and right are shown in Fig. 3. We defined two functional ROIs of the peak voxels in dorsal stream and ventral stream regions using the Marsbar software (http://marsbar.sourceforge.net/) and extracted the mean time-course across activated voxels in each ROI for each participant. Percent signal change was calculated individually for each subject using that subject's fixation activation as baseline and then averaging across subjects. From the results of BOLD signal changes, the activated intensity and voxels of SG were higher than those of ST and SGT in the dorsal and ventral streams of left and right.

\subsection{SGT vs. $S T$}

Similarly, as shown in Fig. 2(b), the SGT compared with the control tasks (ST) also more significantly activated the ventral and dorsal streams, in which the ventral stream had a $19480 \mathrm{~mm}^{3}$ cluster size including the bilateral lingual gyrus (BA18/19, TAL: $-20 /-70 /-3, t=10.65 ;-8 /-74 / 2, t=9.68$; $8 /-72 / 7, t=10.03$ ), fusiform gyrus (BA19, TAL: $-32 /-45 /-8, t=8.92 ; 26 /-63 /-7, t=13.02$ ), 
and posterior parahippocampal gyrus (BA19, TAL: $-20 /-45 /-4, t=8.28 ; 30 /-47 /-6, t=10.49$ ); the dorsal stream had a $5344 \mathrm{~mm}^{3}$ cluster size including the bilateral superior occipital gyrus (BA19, TAL: $-36 /-81 / 17, t=6.87 ; 34 /-80 / 30, t=7.21)$, and right precuneus extending to the right superior parietal lobule (BA7, TAL: $20 /-66 / 47, t=7.29$ ). The results suggest that both ventral and dorsal streams are commonly involved in cognitive processing of statistical graphs, even though a verbal description is added to a statistical graph.

\section{3. $S G v s . S G T$}

To further verify the differences between the SG and SGT, we did the SG compared with the SGT. For the thresholded at $p<0.05$ (corrected), we didn't find any brain activation; for the thresholded at $p<0.001$ (uncorrected), the results showed that only the ventral stream had a $7216 \mathrm{~mm}^{3}$ cluster size including the bilateral lingual gyrus (BA18, TAL: $-18 /-72 /-1, t=6.65 ;-6 /-76 / 2, t=5.51 ; 8 /-74 / 6$, $t=4.60$ ), fusiform gyrus (BA20, TAL: $-32 /-41 /-13, t=4.64 ; 38 /-38 /-18, t=4.34$ ) and posterior parahippocampal gyrus (BA19, TAL: $34 /-28 /-19, t=4.39$ ) was more significantly activated, but no dorsal stream (Fig. 2(c)).

In contrast, we also compared SGT with SG in the same condition ( $p<0.001$, uncorrected), and found only the striate/prestriate cortex (BA17/18) in the occipital lobe were activated, but no dorsal stream, as shown in Fig. 2(d). The results indicate that there are significant differences in ventral stream between SG and SGT, whereas there is no significant difference in dorsal stream. In our study, the text of SGT is mainly the basic description of the items of graphs, which doesn't involve the information analysis (e.g. the prediction of a trend). That is, the text of SGT is more important for extracting the statistical information than for the integration of the information. Thus, the activations in SG vs. SGT should be more involved in the aspects of the perceptual processing relevant the data extraction, which is also consistent with the basic functions of the ventral stream. In contrast, we can conclude that the dorsal stream which was not activated in SG vs. SGT should be more involved in information analysis, which is also according with the basic function of the dorsal stream that is related to the objects' location recognition and spatial analysis.

\section{Discussion}

Many previous studies have implicated that the fusiform gyrus is more involved in object identification and recognition. For example, Jernigan et al. investigated the effects of degrading letter integrity and found increased activity in the fusiform gyrus for both identifying and recognizing degraded words [12]. Moreover, Bar et al. studied the cortical mechanisms associated with object recognition using fMRI and found that fusiform gyrus activation gradually increased with subjective ratings of successful recognition [13].

Attention to global shapes generated stronger activation over the medial occipital cortex relative to local attention [14], and the lingual gyrus was more involved in spatial attention [15]. Previous studies indicated that the lingual gyrus is strongly activated in the integration of local information For example, some studies investigated the processing of the global and local hierarchically organized and found that the lingual gyri were strongly activated during the integration of local and global aspects [16]17]. Similarly, Jin et al. indicated that associative processing with predictive inference increased lingual gyrus activation [18]. Therefore, during statistical graph comprehension, subjects first read the values of various items, involving the integration of various elements (e.g., $x$-axis labels, the height of a correspond- 
ing bar (bar-graphs), the location of data points (line-graphs), or the corresponding y-values) to form a meaningful graph. This processing requires spatial attention and integration of local information, which recruits the lingual gyrus.

The parahippocampal gyrus (PHG) has been traditionally implicated in episodic memory. More recent studies suggest that this region responds more to associative processing, such as object-location associations [19] and contextual associations [20]. Furthermore, studies focusing on binding information showed that during binding information with associations compared with non-binding information, the PHG was more activated [21]. The posterior portion of the PHG is more involved in spatial contexts, whereas the anterior PHG is more involved in nonspatial contexts [22]. In our study, statistical graph comprehension not only requires element recognition, but also needs associations between the object and its specific location. Different from text, the process of extracting information from statistical graphs mainly depends on the spatial-location associations, which involve the processing associated with spatial contexts and episodic memory. Thus, the posterior PHG is more involved in statistical graph comprehension.

Not only the ventral stream was more significantly activated in (SG vs. ST) and (SGT vs. ST), but the dorsal stream including the superior occipital gyrus, precuneus and superior parietal lobule was also activated. It is widely accepted that the dorsal stream is involved in spatial processing. Previous studies have indicated that the superior parietal gyrus is involved in object-location encoding [23] and visual spatial processing [24]. Consistent with these findings, the lesions study also suggested that damage to the region caused visuo-spatial deficits [25]. Moreover, it has been reported that the precuneus, superior parietal lobule and adjacent visual cortical regions (superior occipital gyrus) have functional connectivity [26], and these regions are more involved in spatial information processing. Feeney et al. have proposed that statistical graph comprehension requires relative reasoning [4]. Thus, the precuneus, superior parietal lobule and superior occipital gyrus are mainly involved in the spatial-location related processing during statistical graph comprehension, such as identifying the locations of the elements (e.g., Bar or Line), and their spatial relationships analysis. In addition, the information integration requires the involvement of spatial processing, such as constructing a mental representation, mental rotation of objects, comparing between two adjacent or non-adjacent Bars, as well as processing the relationships between items (including recognizing the maximum and minimum, and sorting the various items).

We also compared SG with SGT, and found that only the ventral stream including the lingual gyrus, fusiform gyrus, and posterior parahippocampal gyrus were activated, but no dorsal stream, which suggest that there are significant differences in ventral stream between SG and SGT, whereas there is no significant difference in dorsal stream. In our study, SGT text is mainly the verbal description of the items of SG, and the SGT comprehension is text-oriented [10|11|27], thus, subjects tend to extract the information from the text first, and then generate graphical perception operation, which lead to the decreased activity in the ventral stream that related to the items recognition and associative memory. Both SG and SGT are the same in the information analysis and integration because SGT text doesn't involve the description of information analysis. Therefore, in both SG and SGT, the comprehension or information integration requires the strong involvement of the dorsal stream that is related to the identifying locations of the items, and their spatial relationships analysis. It may be the reason that there is no significant difference in dorsal stream during SG compared with SGT.

\section{Acknowledgments}

This work is supported by the 973 Program (No. 2014CB744600), the International Science \& Technology Cooperation Program of China (No. 2013DFA32180), the National Natural Science Foundation 
of China (No. 61272345), the Beijing Natural Science Foundation (No. 4132023), the Beijing Outstanding Talent Training Foundation (No. 2014000020124G039), and the Open Research Fund of Beijing Key Lab of Magnetic Resonance Imaging and Brain Informatics (No. 2014CGZC03).

\section{References}

[1] Fry E, editor. A theory of graphs for reading comprehension and writing communication. New Brunswick (NJ): Rutgers University; 1983.

[2] Kosslyn SM. Understanding charts and graphs. Appl Cogn Psychol. 1989; 3: 185-226. doi: 10.1002/acp.2350030302.

[3] Zacks J, Tversky B. Bars and lines: A study of graphic communication. Mem and Cogn. 1999; 27(6): 1073-1079. doi: 10.3758/BF03201236.

[4] Feeney A, Webber L. Analogical representation and graph comprehension. LNCS. 2003; 2733: 212-221. doi: 10.1.1.97. 9774.

[5] Trafton JG, Kirschenbaum SS, Tsuji TL, Miyamoto RT, Ballas JA, Raymond PD. Turning pictures into numbers: extracting and generating information from complex visualizations. Int J Hum-Comput ST. 2000; 53(5): 827-850. doi: 10.1006/ijhc.2000.0419.

[6] Trickett SB, Trafton JG. Toward a comprehensive model of graph comprehension: making the case for spatial cognition. LNCS. 2006; 4045: 286-300. doi: 10.1007/11783183_38.

[7] Mishkin M, Ungerleider LG, Macko KA. Object vision and spatial vision - 2 cortical pathways. Trends Neurosci. 1983; 6: 414-417. doi: 10.1016/0166-2236(83)90190-X.

[8] James TW, Culham J, Humphrey GK, Milner AD, Goodale MA. Ventral occipital lesions impair object recognition but not object-directed grasping: an fMRI study. Brain. 2003; 126: 2463-2475. doi: 10.1093/brain/awg248.

[9] Schindler I, Rice NJ, McIntosh RD, Rossetti Y, Vighetto A, Milner AD. Automatic avoidance of obstacles is a dorsal stream function: evidence from optic ataxia. Nature Neurosci. 2004; 7(7): 779-784. doi: 10.1038/nn1273.

[10] Carpenter PA, Shah P. A model of the perceptual and conceptual processes in graph comprehension. J Exp Psychol Appl. 1998; 4(2): 75-100. doi: 10.1037/1076-898X.4.2.75.

[11] Rayner K, Rotelloa CM, Stewartb AJ, Keira J, Duffy SA. Integrating text and pictorial information: eye movements when looking at print advertisements. J Exp Psychol Appl. 2001; 7(3): 219-226. doi: 10.1037/1076-898X.7.3.219.

[12] Jernigan TL, Ostergaard AL, Law I, Svarer C, Gerlach C, Paulson OB. Brain activation during word identification and word recognition. Neuroimage. 1998; 8(1): 93-105. doi: 10.1006/nimg.1998.0350.

[13] Bar M, Tootell R, Schacter DL, Greve DN, Fischl B, Mendola JD, et al. Cortical mechanisms specific to explicit visual object recognition. Neuron. 2001; 29(2): 529-535. doi: 10.1016/S0896-6273(01)00224-0.

[14] Han SH, Weaver JA, Murray SO, Kang XJ, Yund EW, Woods DL. Modulation of human global local perception by low spatial frequency filtering. Chin Sci Bull. 2003; 48(21): 2336-2339. doi: 10.1360/03wc0221.

[15] Martinez A, Anllo-Vento L, Sereno MI, Frank LR, Buxton RB, Dubowitz DJ, et al. Involvement of striate and extrastriate visual cortical areas in spatial attention. Nat Neurosci. 1999; 2: 364-369. doi: 10.1038/7274.

[16] Fink GR, Marshall JC, Halligan PW, Dolan RJ. Hemispheric asymmetries in global/local processing are modulated by perceptual salience. Neuropsychologia. 1999; 37(1): 31-40. doi: 10.1016/S0028-3932(98)00047-5.

[17] Lux S, Marshall JC, Ritzl A, Weiss PH, Pietrzyk U, Shah NJ, et al. A functional magnetic resonance imaging study of local/global processing with stimulus presentation in the peripheral visual hemifields. Neuroscience. 2004; 124(1): 113-120. doi: 10.1016/j.neuroscience.2003.10.044.

[18] Jin H, Liu HL, Mo L, Fang SY, Zhang JX, Lin CD. Involvement of the left inferior frontal gyrus in predictive inference making. Int J Psychophysiol. 2009; 71(2): 142-148. doi: 10.1016/j.ijpsycho.2008.08.009.

[19] Sommer T, Rose M, Weiller C, Buchel C. Contributions of occipital, parietal and parahippocampal cortex to encoding of object-location associations. Neuropsychologia. 2005; 43(5): 732-743. doi: 10.1016/j.neuropsychologia.2004.08.002.

[20] Bar M, Aminoff E, Ishai A. Famous faces activate contextual associations in the parahippocampal cortex. Cereb Cortex. 2008; 18(6): 1233-1238. doi: 10.1093/cercor/bhm170.

[21] Luck D, Danion JM, Marrer C, Pham BT, Gounot D, Foucher J. The right parahippocampal gyrus contributes to the formation and maintenance of bound information in working memory. Brain Cogn. 2010; 72(2): 255-263. doi: 10.1016/j.bandc.2009.09.009.

[22] Aminoff E, Gronau N, Bar M. The parahippocampal cortex mediates spatial and nonspatial associations. Cereb Cortex. 2007; 17(7): 1493-1503. doi: 10.1093/cercor/bhl078.

[23] Vandenberghe R, Gitelman DR, Parrish TB, Mesulam MM. Functional specificity of superior parietal mediation of spatial shifting. Neuroimage. 2001; 14(3): 661-673. doi: 10.1006/nimg.2001.0860.

[24] Gogos A, Gavrilescu M, Davison S, Searle K, Adams J, Rossell SL, et al. Greater superior than inferior parietal lobule 
activation with increasing rotation angle during mental rotation: An fMRI study. Neuropsychologia. 2010; 48(2): 529535. doi: 10.1016/j.neuropsychologia.2009.10.013.

[25] Sack AT, Kohler A, Bestmann S, Linden D, Dechent P, Goebel R, et al. Imaging the brain activity changes underlying impaired visuospatial judgments: Simultaneous fMRI, TMS, and behavioral studies. Cereb Cortex. 2007; 17(12): 28412852. doi: $10.1093 /$ cercor/bhm013.

[26] Margulies DS, Vincent JL, Kelly C, Lohmann G, Uddin LQ, Biswal BB, et al. Precuneus shares intrinsic functional architecture in humans and monkeys. PNAS. 2009; 106(47): 20069-20074. doi: 10.1073/pnas.0905314106.

[27] Kima HS, Choia MH, Yoona HJ, Kima HJ, Jeounga UH, Parka SJ, et al. Cerebral activation and lateralization due to the cognition of a various driving speed difference: An fMRI study. Biomed Mater Eng. 2014; 24(1): 1133-1139. doi: 10.3233/BME-130913. 